ESAIM: M2AN 47 (2013) 305-315

DOI: $10.1051 / \mathrm{m} 2 \mathrm{an} / 2012033$
ESAIM: Mathematical Modelling and Numerical Analysis

www.esaim-m2an.org

\title{
BOUND STATES OF A CONVERGING QUANTUM WAVEGUIDE
}

\author{
Giuseppe Cardone ${ }^{1}$, Sergei A. Nazarov ${ }^{2}$ and Keijo Ruotsalainen ${ }^{3}$
}

\begin{abstract}
We consider a two-dimensional quantum waveguide composed of two semi-strips of width 1 and $1-\varepsilon$, where $\varepsilon>0$ is a small real parameter, i.e. the waveguide is gently converging. The width of the junction zone for the semi-strips is $1+O(\sqrt{\varepsilon})$. We will present a sufficient condition for the existence of a weakly coupled bound state below $\pi^{2}$, the lower bound of the continuous spectrum. This eigenvalue in the discrete spectrum is unique and its asymptotics is constructed and justified when $\varepsilon \rightarrow 0^{+}$.
\end{abstract}

Mathematics Subject Classification. 35P15, 47A75, 49R50.

Received September 2, 2011. Revised May 14, 2012.

Published online November 23, 2012.

\section{INTRODUCTION}

The spectral properties of the Dirichlet Laplacian in unbounded geometries, especially in two-dimensional tubes, have attracted some attention during the last two decades [1,3-12,14-16] because it provides a simple model for electron motion in quantum wires, i.e., tiny strips of a very pure semiconductor material prepared on a substrate.

The most spectacular manifestation of this kind of mesoscopic physics is the thin films produced on insulating surfaces by embossing or by printing. Their thickness ranges from 10-100 nm which means that the transversal cross-section of such a thin layer contains several atoms only.

The behaviour of an electron in such a structure is, of course, covered by the many-body Schrödinger equation describing its interaction with the atoms (or molecules) including possible impurities. However, due to the small size, high purity and the crystalline structure the mean-free-path of the particle can be two or three orders of magnitude larger than the size of the structure. Hence the electron motion falls in the ballistic regime, as the experimentalists put it. This means that the propagation of the electron is largely undisturbed by the scattering on impurities. Hence we may take a potential which is zero inside the channel and infinite outside. In this case inside the waveguide, the Hamiltonian is just $H=\frac{p^{2}}{2 m^{*}}$ with some effective mass $m^{*}$, which is considerably smaller than the electron mass, and with the boundary conditions that suppress the particle's wave function to

\footnotetext{
Keywords and phrases. Quantum waveguide, spectrum, asymptotics.

1 University of Sannio - Department of Engineering, Piazza Roma, 21, 84100 Benevento, Italy. giuseppe.cardone@unisannio.it

2 Institute of Mechanical Engineering Problems, V.O., Bolshoi pr., 61, 199178 St. Petersburg, Russia.

srgnazarov@yahoo.co.uk

3 University of Oulu - Department of Electrical Engineering, P.O. Box 4500, 90014 Oulu, Finland.

keijo.ruotsalainen@ee.oulu.fi
} 


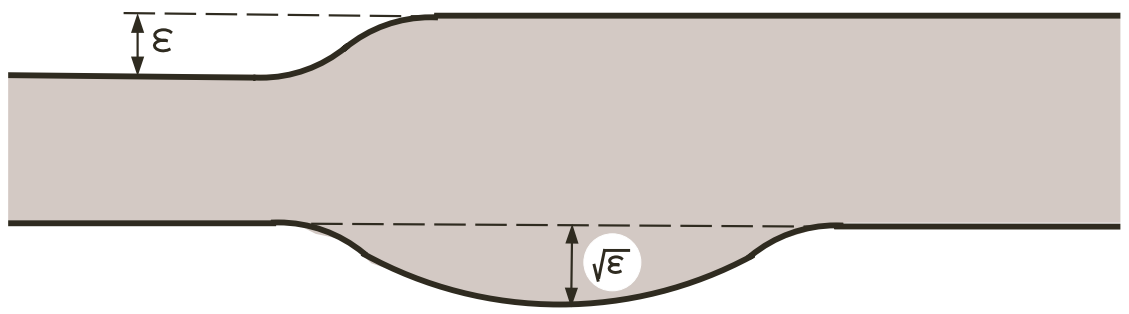

Figure 1. The converging waveguide.

go to zero on the sides of the channel. An interaction term must be added only if the whole structure is placed in an external field.

The investigation of this kind of quantum motion in a fixed subset of the configuration space is usually referred to as quantum waveguides in view its natural analogy to the classical wave motion.

The motivation of our paper stems from a fundamental problem in atomic physics, where matter-field coupling leads to a non-local dressing of the steady states of the Hamiltonian, as well as instability of excited states due to the resonance interaction. Therefore we consider a two-dimensional ballistic quantum waveguide that consists of a resonance cavity with co-axial leads on two sides with different thickness. We show that the quantum waveguide may mimic an atom on a mesoscopic scale. The bound state inside the cavity correspond to the discrete spectrum of an atom. The advantage of our approach is that one can control physical parameters only by changing the geometry of the waveguide.

\section{The PRELIMINARIES AND THE RESUlT}

In this paper, we study the bound state spectra of a two-dimensional quantum waveguide $\Pi^{\varepsilon}$ composed of two semi-strips (see Fig. 1)

$$
\begin{aligned}
& \Pi_{+}=[R,+\infty) \times(0,1), \\
& \Pi_{-}^{\varepsilon}=(-\infty,-R) \times(0,1-\varepsilon)
\end{aligned}
$$

and of the middle part $\Pi_{0}^{\varepsilon}$ of a finite volume. In (2.1) $\varepsilon$ denotes a small positive parameter, i.e. the width $1-\varepsilon$ of $\Pi_{-}^{\varepsilon}$ is smaller than the unit width of $\Pi_{+}$so that $\Pi^{\varepsilon}$ is a converging waveguide. An adequate model of this quantum waveguide refers to the spectral Dirichlet problem for the Laplace operator $\Delta=\nabla \cdot \nabla$

$$
\begin{gathered}
-\Delta u^{\varepsilon}(x)=\lambda^{\varepsilon} u^{\varepsilon}(x), x \in \Pi^{\varepsilon}, \\
u^{\varepsilon}(x)=0, x \in \partial \Pi^{\varepsilon} .
\end{gathered}
$$

It is known that the continuous spectrum $\sigma_{c}^{\varepsilon}$ of the problem $(2.2)$ consists of the ray $\left[\pi^{2},+\infty\right)$ while the discrete spectrum $\sigma_{d}^{\varepsilon}$ of finite total multiplicity may occur in the interval $\left(0, \pi^{2}\right)$. Notice that the lower bound $\lambda_{\dagger}=\pi^{2}$ of $\sigma_{c}=\sigma_{c}^{\varepsilon}$ is defined by the widest semi-strip in (2.1).

Let us assume for a while that the waveguide is given by

$$
\Pi^{\varepsilon}=\left\{x \in \mathbb{R}^{2}: x_{1} \in \mathbb{R}, 0<x_{2}<1-\varepsilon h\left(x_{1}\right)\right\} .
$$

In the case of the local perturbation of the straight strip, namely when the smooth function $h$ in (2.3) vanishes for both $x_{1}>R$ and $x_{1}<-R$, a result given in [11] says that under the condition

$$
\int_{\mathbb{R}} h\left(x_{1}\right) \mathrm{d} x_{1}<0
$$


and for all small $\varepsilon>0$ the discrete spectrum $\sigma_{d}^{\varepsilon}$ contains just one eigenvalue $\lambda_{1}^{\varepsilon}$ and, moreover,

$$
\lambda_{1}^{\varepsilon}=\pi^{2}\left(1-\pi^{2} \varepsilon^{2}\left(\int_{\mathbb{R}} h\left(x_{1}\right) \mathrm{d} x_{1}\right)^{2}\right)+O\left(\varepsilon^{3}\right) .
$$

If $h$ is non-positive everywhere, no smallness assumption on $\varepsilon$ is needed to conclude that $\sigma_{d}^{\varepsilon} \neq \emptyset$.

Note that formulae (2.3) and (2.4) ensure a positive increment in the volume of the waveguide in comparison with the straight strip $\Pi^{0}$. On the contrary, if the perturbation satisfies the condition

$$
\int_{\mathbb{R}} h\left(x_{1}\right) \mathrm{d} x_{1}>0
$$

the volume of the straight waveguide decreases. In this case, the discrete spectrum $\sigma_{d}^{\varepsilon}$ becomes empty for all small $\varepsilon>0$.

In our case, the perturbation is no longer local, because in order to fulfil (2.1) the function $h$ must satisfy the conditions

$$
\begin{aligned}
& h\left(x_{1}\right)=0 \text { for } x_{1}>R \text { but } \\
& h\left(x_{1}\right)=1 \text { for } x_{1}<-R .
\end{aligned}
$$

Intuitively, it is clear that the relation (2.6) provide the infinite decrease in the volume of the waveguide and $\sigma_{d}^{\varepsilon}$ must stay empty. To prove this fact rigorously, we choose a number $R_{\#}>R$ and a function $h_{\#}$ such that

$$
\begin{aligned}
& h_{\#}\left(x_{1}\right)=0 \text { for }\left|x_{1}\right|>R_{\#}, \\
& h_{\#}\left(x_{1}\right) \leq h\left(x_{1}\right) \text { for } x_{1} \in \mathbb{R}, \\
& \int_{\mathbb{R}} h_{\#}\left(x_{1}\right) \mathrm{d} x_{1}>0 .
\end{aligned}
$$

Let us assume that the problem (2.2) in $\Pi^{\varepsilon}$ has an eigenvalue $\lambda_{1}^{\varepsilon} \in\left(0, \pi^{2}\right)$. By extending the corresponding eigenfunction $u_{1}^{\varepsilon} \in H^{2}\left(\Pi^{\varepsilon}\right) \cap H_{0}^{1}\left(\Pi^{\varepsilon}\right)$ (the Sobolev spaces) as zero onto

$$
\Pi_{\#}^{\varepsilon}=\left\{x: x_{1} \in \mathbb{R}, 0<x_{2}<1-\varepsilon h_{\#}\left(x_{1}\right)\right\}
$$

and writing down the minimum principle we obtain

$$
\begin{aligned}
\lambda_{\#}^{\varepsilon} & =\inf _{v^{\varepsilon} \in H^{2}\left(\Pi_{\#}^{\varepsilon}\right) \cap H_{0}^{1}\left(\Pi_{\#}^{\varepsilon}\right)} \frac{\left\|\nabla v^{\varepsilon}\right\|_{L^{2}\left(\Pi_{\#}^{\varepsilon}\right)}^{2}}{\left\|v^{\varepsilon}\right\|_{L^{2}\left(\Pi_{\#}^{\varepsilon}\right)}^{2}} \\
& \leq \frac{\left\|\nabla u_{1}^{\varepsilon}\right\|_{L^{2}\left(\Pi_{\#}^{\varepsilon}\right)}^{2}}{\left\|u_{1}^{\varepsilon}\right\|_{L^{2}\left(\Pi_{\#}^{\varepsilon}\right)}^{2}} \\
& =\frac{\left\|\nabla u^{\varepsilon}\right\|_{L^{2}\left(\Pi^{\varepsilon}\right)}^{2}}{\left\|u^{\varepsilon}\right\|_{L^{2}\left(\Pi^{\varepsilon}\right)}^{2}}=\lambda_{1}^{\varepsilon}<\pi^{2} .
\end{aligned}
$$

Here $H_{0}^{1}\left(\Pi^{\varepsilon}\right)$ is a subspace of functions $v^{\varepsilon} \in H^{1}\left(\Pi^{\varepsilon}\right)$ which vanish on $\partial \Pi^{\varepsilon}$, and $\left\|v^{\varepsilon}\right\|_{L^{2}\left(\Pi^{\varepsilon}\right)}$ denotes the natural norm in the Lebesgue space $L^{2}\left(\Pi^{\varepsilon}\right)$.

By Theorem 10.2.1 in [2], the discrete spectrum of the problem in $\Pi_{\#}^{\varepsilon}$ is not empty due to the inequality (2.8), which contradicts to the above mentioned result in [11] due to the last condition in (2.7), cf. (2.5). Therefore the discrete spectrum is empty $\left(\sigma_{d}^{\varepsilon}=\varnothing\right)$ in the quantum waveguide $\Pi^{\varepsilon}$ given by $(2.3)$ and $(2.6)$.

In other words, in the case

$$
\Pi_{0}^{\varepsilon} \subset(-R, R) \times(-C \varepsilon, 1+C \varepsilon)
$$


with some constant $C>0$ the discrete spectrum of problem (2.2) is always empty since any local perturbation of magnitude $O(\varepsilon)$ cannot compensate for the infinite decrease of the waveguide volume caused by shrinking the semi-strip $\Pi_{-}^{\varepsilon}$.

On the other hand, according to result by Jones [21], $\sigma_{d}^{\varepsilon} \neq \varnothing$ provided the middle part $\Pi_{0}^{\varepsilon}$ is sufficiently big. In this case the principal eigenvalue of the Dirichlet problem in $\Pi_{0}^{\varepsilon}$ is less than $\pi^{2}$. This fact is again confirmed by applying the minimum principle similarly to (2.8). Hence the following question is addressed in this paper: what is the size of $\Pi_{0}^{\varepsilon}$ to guarantee the existence of an eigenvalue inside the interval $\left(0, \pi^{2}\right)$ ? In this paper we will show that the width $1+O\left(\varepsilon^{1 / 2}\right)$ is sufficient.

To be more precise, we assume that the waveguide is of the form

$$
\Pi^{\varepsilon}=\left\{x: x_{1} \in \mathbb{R},-\sqrt{\varepsilon} H_{-}\left(x_{1}\right)<x_{2}<1-\varepsilon h\left(x_{1}\right)+\sqrt{\varepsilon} H_{+}\left(x_{1}\right)\right\}
$$

and, in addition to $(2.6)$,

$$
H_{ \pm}\left(x_{1}\right)=0 \text { for }\left|x_{1}\right|>R, \quad H_{ \pm} \text {are smooth. }
$$

In what follows we will prove the following assertion.

Theorem 2.1. If

$$
\bar{H}:=\int_{\mathbb{R}} H\left(x_{1}\right) \mathrm{d} x_{1}>\frac{1}{\pi \sqrt{2}}, \quad H=H_{+}+H_{-}
$$

then the problem (2.2) in the waveguide (2.9) has a unique eigenvalue $\lambda_{1}^{\varepsilon}$ inside the interval $\left(0, \pi^{2}\right)$. The eigenvalue $\lambda_{1}^{\varepsilon}$ has the asymptotic behavior

$$
\lambda_{1}^{\varepsilon}=\pi^{2}-\varepsilon \frac{\left(2 \pi^{2} \bar{H}^{2}-1\right)^{2}}{4 \bar{H}^{2}}+O\left(\varepsilon^{5 / 4}\right)
$$

as $\varepsilon \rightarrow 0^{+}$.

We emphasize that the enlarging of the waveguide $\Pi_{0}^{\varepsilon}$ yields the diminishing of the eigenvalue so that the discrete spectrum remains non-empty if (2.6), (2.11) hold true and

$$
\Pi_{0}^{\varepsilon} \supset\left\{x:\left|x_{1}\right|<R,-\sqrt{\varepsilon} H_{-}\left(x_{1}\right)<x_{2}<1-\varepsilon h\left(x_{1}\right)+\sqrt{\varepsilon} H_{+}\left(x_{1}\right)\right\} .
$$

It is remarkable that the profile function $h$ is not involved in the main asymptotic term of (2.12).

Let us outline several results about the local perturbations of the cylindrical quantum waveguides. First of all, the above mentioned result in [11] was complemented in [18] by demonstrating that, in the waveguide of the form given by (2.3) and (2.6) having $h$ with vanishing average, both the existence and non-existence of an eigenvalue in $\left(0, \pi^{2}\right)$ may occur. In the paper [10], the asymptotic behaviour of the eigenvalue, if it exists, was constructed.

In $[19,20]$ the perturbation of the Laplace operator by a small second-order differential operator was considered in a waveguide in $\mathbb{R}^{n}$. Such perturbations, after an appropriate change of coordinates, cover a regular perturbation of the boundary as well as a gently twisting of the cylindrical waveguide in $\mathbb{R}^{3}$ (notice that in the last case the discrete spectrum is empty, see [20]). The existence of eigenvalues below the continuous spectrum in gently curved quantum waveguides in $\mathbb{R}^{n}$ was proved in [17] and the asymptotic expansion was constructed. Cranked waveguides were studied in [1,27].

All the above cited papers consider quantum waveguides which, outside a big ball, have constant cross-sections and it looks that the present paper for the first time considers a waveguide with the different width of the outlets into infinity. Also the methods applied here are different from the ones in the cited publications. To derive the representation (2.12) in Section 3, we use the asymptotic analysis [25] although we find out another argument to compute the correction term $O(\varepsilon)$. The justification scheme in Section 4 is based on the spectral measure technique (see [2], Chap. 5.6). 


\section{The FORMAL ASYMPtotic ANALYSis}

We search for an eigenvalue of the problem (2.2) in the form

$$
\lambda^{\varepsilon}=\pi^{2}-\varepsilon \beta+\widetilde{\lambda}^{\varepsilon}, \quad \beta>0 .
$$

The asymptotic behavior of the corresponding eigenfunction $u^{\varepsilon} \in H^{2}\left(\Pi^{\varepsilon}\right) \cap H_{0}^{1}\left(\Pi^{\varepsilon}\right)$, as $x_{1} \rightarrow \pm \infty$, is described by the following main terms:

$$
\begin{aligned}
& c_{+}^{\varepsilon} \sin \left(\pi x_{2}\right) \exp \left(-x_{1} \sqrt{\pi^{2}-\lambda^{\varepsilon}}\right) \text { in } \Pi_{+}^{\varepsilon}, \\
& c_{-}^{\varepsilon} \sin \left(\pi(1-\varepsilon)^{-1} x_{2}\right) \exp \left(x_{1} \sqrt{\pi^{2}(1-\varepsilon)^{-2}-\lambda^{\varepsilon}}\right), \text { in } \Pi_{-}^{\varepsilon} .
\end{aligned}
$$

Inside a finite part of the waveguide, the expressions in (3.2) decompose as

$$
\begin{aligned}
& \left(c_{+}^{0}+\varepsilon^{1 / 2} c_{+}^{\prime}+\ldots\right) \sin \left(\pi x_{2}\right) \exp \left(-x_{1} \varepsilon^{1 / 2} \beta^{1 / 2}+\ldots\right) \\
& \quad=\sin \left(\pi x_{2}\right)\left(c_{+}^{0}+\varepsilon^{1 / 2}\left(c_{+}^{\prime}-c_{+}^{0} \varepsilon^{1 / 2} \beta^{1 / 2} x_{1}\right)+\ldots\right) \\
& \left(c_{-}^{0}+\varepsilon^{1 / 2} c_{-}^{\prime}+\ldots\right)\left(\sin \left(\pi x_{2}\right)+\ldots\right) \exp \left(x_{1} \varepsilon^{1 / 2}\left(2 \pi^{2}+\beta\right)^{1 / 2}+\ldots\right) \\
& =\sin \left(\pi x_{2}\right)\left(c_{-}^{0}+\varepsilon^{1 / 2}\left(c_{-}^{\prime}+c_{-}^{0} \varepsilon^{1 / 2}\left(2 \pi^{2}+\beta\right)^{1 / 2} x_{1}\right)+\ldots\right) .
\end{aligned}
$$

Here and in the sequel the dots stand for lower order terms inessential for our formal asymptotic analysis. We thus make the asymptotic ansatz

$$
u^{\varepsilon}(x)=u^{0}(x)+\varepsilon^{1 / 2} u^{\prime}(x)+\cdots
$$

for the corresponding eigenfunction. Setting $\varepsilon=0$ in equations (2.2) and (2.9) leads to the following homogeneous $\left(g_{p}=0\right)$ limit problem in the straight strip $\Pi^{0}=\mathbb{R} \times(0,1)$ :

$$
\begin{aligned}
-\Delta u^{0}(x) & =\pi^{2} u^{0}(x), x \in \Pi^{0} \\
u^{0}\left(x_{1}, p\right) & =g_{p}\left(x_{1}\right), x_{1} \in \mathbb{R}, p=0,1 .
\end{aligned}
$$

This problem has two explicit solutions

$$
u_{0}^{0}(x)=\sin \left(\pi x_{2}\right), u_{1}^{0}(x)=x_{2} \sin \left(\pi x_{2}\right) .
$$

Immediately, we conclude that $u^{0}(x)$ in (3.4) satisfies the homogeneous problem (3.5). Considering the expansions (3.3) as representations of the right-hand side in (3.5), we detect that $u^{0}(x) \rightarrow c_{ \pm}^{0} \sin \left(\pi x_{2}\right)$, when $x_{1} \rightarrow \pm \infty$. Therefore, we conclude that

$$
c_{+}^{0}=c_{-}^{0}=1, u^{0}(x)=u_{0}^{0}(x)=\sin \left(\pi x_{2}\right) .
$$

Notice that we have fixed $c_{ \pm}^{0}=1$ in order to normalize the eigenfunctions.

The function $u^{0}$ satisfies the Helmholtz equation in $\Pi^{\varepsilon}$ with the parameter $\lambda^{0}=\pi^{2}$ but leaves the discrepancies

$$
\begin{aligned}
& \left.u^{0}(x)\right|_{\varpi_{0}^{\varepsilon}}=-\varepsilon^{1 / 2} \pi H_{-}\left(x_{1}\right)+O(\varepsilon), \\
& \left.u^{0}(x)\right|_{\varpi_{1}^{\varepsilon}}=-\varepsilon^{1 / 2} \pi H_{+}\left(x_{1}\right)+O(\varepsilon)
\end{aligned}
$$


in the boundary conditions on the upper and lower parts

$$
\varpi_{0}^{\varepsilon}=\left\{x_{2}=-\varepsilon^{1 / 2} H_{-}\left(x_{1}\right)\right\}
$$

and

$$
\varpi_{1}^{\varepsilon}=\left\{x_{2}=1+\varepsilon^{1 / 2} H_{+}\left(x_{1}\right)-\varepsilon h\left(x_{1}\right)\right\}
$$

of the curved strip $\Pi^{\varepsilon}$. In (3.8) we have used the Taylor formula. Thus, the correction term $u^{\prime}$ intended to compensate for the discrepancies in (3.8) has to satisfy the problem (3.5) with the right-hand sides

$$
g_{0}\left(x_{1}\right)=\pi H_{-}\left(x_{1}\right), \quad g_{1}\left(x_{1}\right)=\pi H_{+}\left(x_{1}\right) .
$$

Moreover, extracting from (3.3) the multiplier on $\varepsilon^{1 / 2}$, we impose the following conditions at infinity

$$
\begin{aligned}
& u^{\prime}(x)=\sin \left(\pi x_{2}\right)\left(c_{-}^{\prime}+c_{-}^{0}\left(2 \pi^{2}+\beta\right)^{1 / 2} x_{1}\right)+\cdots, \text { as } x_{1} \rightarrow-\infty, \\
& u^{\prime}(x)=\sin \left(\pi x_{2}\right)\left(c_{+}^{\prime}-c_{+}^{0} \beta^{1 / 2} x_{1}\right)+\cdots, \text { as } x_{1} \rightarrow+\infty .
\end{aligned}
$$

By the general theory, the homogeneous problem (3.5) has just two linearly independent solutions. Hence, according to the theory of elliptic problems in domains with cylindrical outlets into infinity (see [22-24] and [28], Chap. 2.5), the problem (3.5) with the asymptotic behaviour (3.9) admits a solution if and only if two compatibility conditions are fulfilled.

The first one can be derived in the following way ( $c f$. [23] and [28], Sect. 5.3). We insert $u^{\prime}$ and $u_{0}^{0}$ into the Green formula on the truncated strip $\Pi^{0}(T)=(-T, T) \times(0,1)$ and let $T \rightarrow+\infty$. Taking into account the first formula in (3.3), we obtain

$$
\begin{aligned}
0= & \lim _{T \rightarrow+\infty} \int_{\Pi^{0}(T)}\left(u_{0}^{0}\left(x_{2}\right)\left(\Delta+\pi^{2}\right) u^{\prime}(x)-u^{\prime}(x)\left(\Delta+\pi^{2}\right) u_{0}^{0}\left(x_{2}\right)\right) \mathrm{d} x \\
= & \lim _{T \rightarrow+\infty}\left(\int_{0}^{1}\left(u_{0}^{0}\left(x_{2}\right) \partial_{1} u^{\prime}\left(T, x_{2}\right)-u_{0}^{0}\left(x_{2}\right) \partial_{1} u^{\prime}\left(-T, x_{2}\right)\right) \mathrm{d} x_{2}\right. \\
& \left.+\int_{-\infty}^{+\infty}\left(u^{\prime}\left(x_{1}, 0\right) \partial_{2} u_{0}^{0}(0)-u^{\prime}\left(x_{1}, 1\right) \partial_{2} u_{0}^{0}(1)\right) \mathrm{d} x_{1}\right) \\
= & -\frac{1}{2} \beta^{1 / 2}-\frac{1}{2}\left(2 \pi^{2}+\beta\right)^{1 / 2}+\pi^{2} \int_{\mathbb{R}} H\left(x_{1}\right) \mathrm{d} x_{1} .
\end{aligned}
$$

Recall that $H=H_{+}+H_{-}$, i.e. $\varepsilon^{1 / 2} \int_{\mathbb{R}} H\left(x_{1}\right) \mathrm{d} x_{1}$ is the main asymptotic term of the volume increment in the waveguide (2.9).

To get the second compatibility condition, one deals with the functions $u^{\prime}$ and $u^{0}$ in the same way as in (3.10). As a result one obtains the following relation:

$$
c_{+}^{\prime}-c_{-}^{\prime}=2 \pi^{2} \int_{\mathbb{R}} x_{1} H\left(x_{1}\right) \mathrm{d} x_{1} .
$$

The equation

$$
\beta^{1 / 2}+\left(2 \pi^{2}+\beta\right)^{1 / 2}=2 \pi^{2} \bar{H}:=2 \pi^{2} \int_{\mathbb{R}} H\left(x_{1}\right) \mathrm{d} x_{1}
$$

inherited from (3.10), has the positive root

$$
\beta^{1 / 2}=\frac{2 \pi^{2} \bar{H}^{2}-1}{2 \bar{H}}
$$

if and only if the requirement (2.11) is satisfied. The correction term $\varepsilon \beta$ in the eigenvalue asymptotics (3.1) is found out from the condition on the existence of the eigenfunction correction term $u^{\prime}$ in (3.4) whose behaviour (3.9) at infinity is matched with decompositions (3.3). 


\section{Justification of the ASYmptotics}

The problem (2.2) gives rise to an unbounded self-adjoint and positive definite operator $A^{\varepsilon}$ in $L^{2}\left(\Pi^{\varepsilon}\right)$ with the domain

$$
\mathcal{D}\left(A^{\varepsilon}\right)=H^{2}\left(\Pi^{\varepsilon}\right) \cap H_{0}^{1}\left(\Pi^{\varepsilon}\right) .
$$

By spectral theorem (see for example [2], Thm. 6.1.1) this operator generates a spectral measure $E_{A^{\varepsilon}}$, which in turn associates to each $U^{\varepsilon} \in L^{2}\left(\Pi^{\varepsilon}\right)$ the scalar measure $\mu_{U^{\varepsilon}}=\left(E_{A^{\varepsilon}} U^{\varepsilon}, U^{\varepsilon}\right)_{\Pi^{\varepsilon}}$ on $\mathbb{R}$. In the sequel, we need the following formulae, which can be found out in the Proof of Theorem 6.1.1 in [2]:

$$
\left\|U^{\varepsilon}\right\|_{L^{2}\left(\Pi^{\varepsilon}\right)}^{2}=\int_{\mathbb{R}} \mathrm{d} \mu_{U^{\varepsilon}}(t)
$$

for $U^{\varepsilon} \in L^{2}\left(\Pi^{\varepsilon}\right)$ and

$$
\left\|A^{\varepsilon} U^{\varepsilon}-\Lambda^{\varepsilon} U^{\varepsilon}\right\|_{L^{2}\left(\Pi^{\varepsilon}\right)}^{2}=\int_{\mathbb{R}}\left(t-\Lambda^{\varepsilon}\right)^{2} \mathrm{~d} \mu_{U^{\varepsilon}}(t)
$$

for $U^{\varepsilon} \in \mathcal{D}\left(A^{\varepsilon}\right), \Lambda^{\varepsilon} \in \mathbb{R}$.

Our justification relies upon the following arguments. First, we fix $\Lambda^{\varepsilon}=\pi^{2}-\varepsilon \beta$ with $\beta>0$ satisfying the equation (3.12). Secondly, we assume that the interval

$$
\Upsilon^{\varepsilon}=\left(\Lambda^{\varepsilon}-K \varepsilon^{5 / 4}, \Lambda^{\varepsilon}+K \varepsilon^{5 / 4}\right)
$$

is free of the essential spectrum of $A^{\varepsilon}$, where $K>0$ is some constant. Notice that $\Upsilon^{\varepsilon} \subset\left(0, \pi^{2}\right)$ for a sufficiently small $\varepsilon>0$. Therefore, $\Upsilon^{\varepsilon}$ may include only the discrete spectrum $\sigma_{d}^{\varepsilon}$ of $A^{\varepsilon}$ of the problem (2.2). Then, for any $U^{\varepsilon} \in \mathcal{D}\left(A^{\varepsilon}\right)$, we deduce by (4.1) and (4.2), that

$$
\begin{aligned}
\left\|A^{\varepsilon} U^{\varepsilon}-\Lambda^{\varepsilon} U^{\varepsilon}\right\|_{L^{2}\left(\Pi^{\varepsilon}\right)}^{2} & =\int_{\mathbb{R} \backslash \Upsilon^{\varepsilon}}\left(t-\Lambda^{\varepsilon}\right)^{2} \mathrm{~d} \mu_{U^{\varepsilon}}(t) \\
& \geq K^{2} \varepsilon^{5 / 2} \int_{\mathbb{R} \backslash \Upsilon^{\varepsilon}} \mathrm{d} \mu_{U^{\varepsilon}}(t) \\
& =K^{2} \varepsilon^{5 / 2} \int_{\mathbb{R}} \mathrm{d} \mu_{U^{\varepsilon}}(t) \\
& =K^{2} \varepsilon^{5 / 2}\left\|U^{\varepsilon}\right\|_{L^{2}\left(\Pi^{\varepsilon}\right)^{2}}
\end{aligned}
$$

Next we construct a function $U^{\varepsilon} \in \mathcal{D}\left(A^{\varepsilon}\right)$ satisfying the inequalities

$$
\begin{aligned}
\left\|A^{\varepsilon} U^{\varepsilon}-\Lambda^{\varepsilon} U^{\varepsilon}\right\|_{L^{2}\left(\Pi^{\varepsilon}\right)} & =\left\|\left(\Delta+\pi^{2}-\varepsilon \beta\right) U^{\varepsilon}\right\|_{L^{2}\left(\Pi^{\varepsilon}\right)} \leq C \varepsilon, \\
\left\|U^{\varepsilon}\right\|_{L^{2}\left(\Pi^{\varepsilon}\right)} & \geq c \varepsilon^{-1 / 4}
\end{aligned}
$$

where $c$ and $C$ are some positive constants. If we now fix $K<(C / c)^{1 / 2}$, the inequality (4.4) contradicts to (4.5) and, thus, the interval (4.3) contains an eigenvalue, which certainly has the asymptotic form (2.12).

The last argument, to conclude the statement of Theorem 2.1, uses the following assertion:

Lemma 4.1. Let $w_{1}^{\varepsilon}$ be the principal eigenmode in the mixed boundary value problem

$$
\begin{aligned}
-\Delta w^{\varepsilon}(x) & =\tau^{\varepsilon} w^{\varepsilon}(x), & & x \in \Pi_{0}^{\varepsilon}, \\
w^{\varepsilon}(x) & =0, & & x \in \partial \Pi_{0}^{\varepsilon} \cap \partial \Pi^{\varepsilon}, \\
\partial_{1} w^{\varepsilon}\left( \pm R, x_{2}\right) & =0, & & x_{2} \in(0,1) .
\end{aligned}
$$


Then any function $u^{\varepsilon} \in H_{0}^{1}\left(\Pi^{\varepsilon}\right)$ satisfying the orthogonality condition

$$
\int_{\Pi_{0}^{\varepsilon}} w_{1}^{\varepsilon}(x) u^{\varepsilon}(x) \mathrm{d} x=0
$$

satisfies the estimate

$$
\left\|u^{\varepsilon}\right\|_{L^{2}\left(\Pi^{\varepsilon}\right)} \leq \pi^{-1}\left\|\nabla u^{\varepsilon}\right\|_{L^{2}\left(\Pi^{\varepsilon}\right)} .
$$

Note that, due to the Max-Min principle ( $c f$. [2], Thm. 10.2.2), the validity of inequality (4.8) under only one orthogonality condition guarantees that the interval $\left(0, \pi^{2}\right)$ cannot include two eigenvalues of the problem (2.2).

Proof. When $\varepsilon=0$, the middle part $\Pi_{0}^{\varepsilon}$ of waveguide $(2.9)$ becomes the rectangle $(-R, R) \times(0,1)$. The problem (4.6) with $\varepsilon=0$ has the eigenvalues

$$
\tau_{1}^{0}=\pi^{2}, \quad \tau_{2}^{0}=\min \left\{4 \pi^{2}, \pi^{2}\left(1+(2 R)^{-2}\right)\right\} .
$$

A simple asymptotic analysis (cf. [26]) of the problem (4.6) delivers the convergence

$$
\tau_{1}^{\varepsilon} \rightarrow \tau_{1}^{0}, \quad \tau_{2}^{\varepsilon} \rightarrow \tau_{2}^{0}>\pi^{2} \quad \text { as } \varepsilon \rightarrow 0^{+} .
$$

Thus, the orthogonality condition (4.7) provides the Poincaré inequality

$$
\left\|\nabla u^{\varepsilon}\right\|_{L^{2}\left(\Pi_{0}^{\varepsilon}\right)}^{2} \geq \tau_{2}^{\varepsilon}\left\|u^{\varepsilon}\right\|_{L^{2}\left(\Pi_{0}^{\varepsilon}\right)}^{2}
$$

while the factor $\tau_{2}^{\varepsilon}$ on the right is bigger than $\pi^{2}$ for a small $\varepsilon>0$. To obtain (4.8), we make use of the Dirichlet condition on the lateral sides of the semi-strips (2.1) and add to (4.9) two Friedrichs' inequalities

$$
\begin{aligned}
& \left\|\partial_{2} u^{\varepsilon}\right\|_{L^{2}\left(\Pi_{+}\right)}^{2} \geq \pi^{2}\left\|u^{\varepsilon}\right\|_{L^{2}\left(\Pi_{+}\right)}^{2}, \\
& \left\|\partial_{2} u^{\varepsilon}\right\|_{L^{2}\left(\Pi_{-}^{\varepsilon}\right)}^{2} \geq \pi^{2}(1-\varepsilon)^{-2}\left\|u^{\varepsilon}\right\|_{L^{2}\left(\Pi_{-}^{\varepsilon}\right)}^{2} .
\end{aligned}
$$

Remark 4.2. According to [25], Chapter 5.9, the asymptotic formula for the principal eigenvalue in problem (4.6) reads as

$$
\tau_{1}^{\varepsilon}=\pi^{2}-\varepsilon^{1 / 2} \frac{\pi^{2}}{R^{1 / 2}} \int_{\mathbb{R}} H\left(x_{1}\right) \mathrm{d} x_{1}+O(\varepsilon) .
$$

Thus, the same argument as in the Proof of Lemma 4.1, owing to (4.10), maintains the absence of eigenvalues in the interval $\left(0, \pi^{2}\right)$ in the evident case $\bar{H}<0$ only. The authors do not know any information on the discrete spectrum for $\bar{H} \in[0, \sqrt{2} / \pi]$.

To prove our theorem, it suffices to construct a function $U^{\varepsilon} \in \mathcal{D}\left(A^{\varepsilon}\right)$ satisfying the mixed boundary value problem (4.6). To this end, we recall the asymptotic decompositions (3.3) and (3.4). From their ingredients, we construct a global approximation $U^{\varepsilon}$ to the eigenfunction $u_{1}^{\varepsilon}$.

First, we extend $u^{\prime}$ smoothly over a neighborhood of $\overline{\Pi_{0}^{\varepsilon}}$, so that the terms of the asymptotic ansatz (3.4) are defined in $\Pi^{\varepsilon}$. Then we construct a function which compensates for the discrepancies of the sum $u^{0}+\varepsilon^{1 / 2} u^{\prime}$ in the boundary conditions on the lower and upper parts $\varpi_{0}^{\varepsilon}, \varpi_{1}^{\varepsilon}$ of the curved strip (2.9). On the lower part $\varpi_{0}^{\varepsilon}$, we have

$$
\begin{aligned}
\left.\left(u^{0}(x)+\varepsilon^{1 / 2} u^{\prime}(x)\right)\right|_{\varpi_{0}^{\varepsilon}}= & u^{0}\left(x_{1},-\varepsilon^{1 / 2} H_{-}\left(x_{1}\right)\right)+\varepsilon^{1 / 2} g_{0}\left(x_{1}\right)+\varepsilon^{1 / 2}\left(u^{\prime}\left(x_{1},-\varepsilon^{1 / 2} H_{-}\left(x_{1}\right)\right)-g_{0}\left(x_{1}\right)\right) \\
= & u^{0}\left(x_{1},-\varepsilon^{1 / 2} H_{-}\left(x_{1}\right)\right)-u^{0}\left(x_{1}, 0\right)+\varepsilon^{1 / 2} H_{-}\left(x_{1}\right) \partial_{2} u^{0}\left(x_{1}, 0\right) \\
& +\varepsilon^{1 / 2}\left(u^{\prime}\left(x_{1},-\varepsilon^{1 / 2} H_{-}\left(x_{1}\right)\right)-u^{0}\left(x_{1}, 0\right)\right) \\
= & O(\varepsilon) .
\end{aligned}
$$


This discrepancy is compensated by a function $\widehat{u}_{0}^{\varepsilon}$ with a support in the neighborhood of the line segment $\left\{x: x_{2}=0,\left|x_{1}\right| \leq R\right\}$ in such a way that

$$
\Delta \widehat{u}_{0}^{\varepsilon}(x)+\left(\pi^{2}-\varepsilon \beta\right) \widehat{u}_{0}^{\varepsilon}(x)=O(\varepsilon) .
$$

A similar discrepancy on $\varpi_{1}^{\varepsilon}$ does not have a compact support because of the shrinking of $\Pi_{-}^{\varepsilon}$. To avoid this difficulty, we employ the asymptotic structure in the method of the compound expansions (see [25], Chap. 2) using the cut-off functions $0 \leq \chi_{ \pm} \leq 1$ such that

$$
\chi_{ \pm}\left(x_{1}\right)= \begin{cases}1 & \text { for } \pm x_{1}>R+1 \\ 0 & \text { for } \mp x_{1}<R .\end{cases}
$$

Namely, we set

$$
\begin{aligned}
U^{\varepsilon}(x)= & \widetilde{u}^{0}(x)+\varepsilon^{1 / 2} \widetilde{u}^{\prime}(x)+\widehat{u}^{\varepsilon}(x)+\chi_{+}\left(x_{1}\right)\left(1+\varepsilon^{1 / 2} c_{+}^{\prime}\right) \sin \left(\pi x_{2}\right) \exp \left(-x_{1} \sqrt{\pi^{2}-\Lambda^{\varepsilon}}\right) \\
& +\chi_{-}\left(x_{1}\right)\left(1+\varepsilon^{1 / 2} c_{-}^{\prime}\right) \sin \left(\pi(1-\varepsilon)^{-1} x_{2}\right) \exp \left(x_{1} \sqrt{\pi^{2}(1-\varepsilon)^{-2}-\Lambda^{\varepsilon}}\right)
\end{aligned}
$$

where $\widetilde{u}^{\prime}$ is the exponentially decaying remainder in the decomposition (3.9),

$$
\begin{aligned}
& \tilde{u}^{0}(x)=u^{0}(x)-\sum_{ \pm} \chi_{ \pm}\left(x_{1}\right) \sin \left(\pi x_{2}\right), \\
& \left.\widetilde{u}^{\prime}(x)=u^{\prime}(x)-\chi_{+}\left(x_{1}\right)\left(c_{+}^{\prime}-\beta^{1 / 2} x_{1}\right)+\chi_{-}\left(x_{1}\right)\left(c_{-}^{\prime}-\left(2 \pi^{2}+\beta\right)^{1 / 2} x_{1}\right)\right) \times \sin \left(\pi x_{2}\right),
\end{aligned}
$$

and the coefficients $c_{ \pm}^{\prime}$ in (3.3) are fixed under the restriction (3.11). Note that $\widetilde{u}^{0}(x)=0$ for $\left|x_{1}\right|>R+1$.

The function $\widehat{u}^{\varepsilon}$ compensates for the discrepancy left by the sum $\widetilde{u}^{0}+\varepsilon^{1 / 2} \widetilde{u}^{\prime} \in H^{2}\left(\Pi^{\varepsilon}\right)$ and, similarly to (4.11), it is chosen such that

$$
\left\|\Delta \widehat{u}^{\varepsilon}+\left(\pi^{2}-\varepsilon \beta\right) \widehat{u}^{\varepsilon}\right\|_{L^{2}\left(\Pi^{\varepsilon}\right)} \leq c \varepsilon .
$$

We write (4.13) as

$$
U^{\varepsilon}(x)=\widetilde{u}^{0}+\varepsilon^{1 / 2} \widetilde{u}^{\prime}+\widehat{u}^{\varepsilon}+\chi_{+} V_{+}^{\varepsilon}+\chi_{-} V_{-}^{\varepsilon}
$$

and observe that the functions $V_{ \pm}^{\varepsilon}$ satisfy the Helmholtz equation with the parameter $\pi^{2}-\varepsilon \beta$. Then we obtain

$$
\left(\Delta+\pi^{2}-\varepsilon \beta\right) U^{\varepsilon}=\left(\Delta+\pi^{2}\right)\left(\widetilde{u}^{0}+\varepsilon^{1 / 2} \widetilde{u}^{\prime}\right)+\sum_{ \pm}\left[\Delta, \chi_{ \pm}\right] V_{ \pm}^{\varepsilon}-\varepsilon \beta\left(\widetilde{u}^{0}+\varepsilon^{1 / 2} \widetilde{u}^{\prime}\right)+\left(\Delta+\pi^{2}-\varepsilon \beta\right) \widehat{u}^{\varepsilon}
$$

where $\left[\Delta, \chi_{ \pm}\right]$is the commutator of $\Delta$ and $\chi_{ \pm}$:

$$
\left[\Delta, \chi_{ \pm}\right] V_{ \pm}^{\varepsilon}=2 \nabla \chi_{ \pm} \cdot \nabla V_{ \pm}^{\varepsilon}+\chi_{ \pm} \Delta V_{ \pm}^{\varepsilon}
$$

Since functions (4.17) have supports in the squares

$$
\left\{x: \pm x_{1} \in[R, R+1], x_{2} \in[0,1]\right\},
$$

we can use the Taylor formulae (3.3). Thus one can see that $L^{2}$-norms of the differences between functions (4.17) and

$$
\begin{aligned}
& {\left[\Delta, \chi_{+}\right]\left(1+\varepsilon^{1 / 2}\left(c_{+}^{\prime}-\varepsilon^{1 / 2} \beta^{1 / 2} x_{1}\right)\right),} \\
& {\left[\Delta, \chi_{-}\right]\left(1+\varepsilon^{1 / 2}\left(c_{-}^{\prime}-\varepsilon^{1 / 2}(2+\beta)^{1 / 2} x_{1}\right)\right)}
\end{aligned}
$$


are just of the order $O(\varepsilon)$. After the substitution $(4.17) \longmapsto(4.18)$, we obtain

$$
\psi^{\varepsilon}=\left(\Delta+\pi^{2}\right)\left(\widetilde{u}^{0}+\varepsilon^{1 / 2} \widetilde{u}^{\prime}\right)+\sum_{ \pm}\left[\Delta, \chi_{ \pm}\right] V_{ \pm}^{\varepsilon} \longmapsto\left(\Delta+\pi^{2}\right)\left(u^{0}+\varepsilon^{1 / 2} u^{\prime}\right) .
$$

Note that $\left(\Delta+\pi^{2}\right) u^{0}=0$, while $\left(\Delta+\pi^{2}\right) u^{\prime}$ vanishes inside $\Pi^{0}$ and equals to $O(\rho)$ in $\Pi^{\varepsilon} \backslash \Pi^{0}$, where $\rho$ is the distance to the sides of the strip $\Pi^{0}$. As a result of this consideration, we obtain

$$
\left\|\psi^{(\varepsilon)}\right\|_{L^{2}\left(\Pi^{\varepsilon}\right)} \leq c \varepsilon
$$

Together with the obvious estimate

$$
\left\|\varepsilon \beta\left(\widetilde{u}^{0}+\varepsilon^{1 / 2} \widetilde{u}^{\prime}\right)\right\|_{L^{2}\left(\Pi^{\varepsilon}\right)} \leq c \varepsilon
$$

the inequalities (4.15) and (4.19) guarantees the first inequality in (4.5) which completes our Proof of Theorem 2.1.

We observe that the remainder order can be shown to be $\varepsilon^{3}$, but for this we need to construct low-order terms and we do not show this in the paper although the construction and justification scheme remains the same.

\section{REFERENCES}

[1] Y. Avishai, D. Bessis, B.G. Giraud and G. Mantica, Quantum bound states in open geometries. Phys. Rev. B 44 (1991) 8028-8034.

[2] M.Sh. Birman and M.Z. Solomjak, Spectral theory of selfadjoint operators in Hilbert space. Translated from the 1980 Russian original by S. Khrushchëv and V. Peller. Math. Appl. (Soviet Series). D. Reidel Publishing Co., Dordrecht (1987).

[3] D. Borisov, R. Bunoiu and G. Cardone, On a waveguide with frequently alternating boundary conditions: homogenized Neumann condition. Ann. Henri Poincaré 11 (2010) 1591-1627.

[4] D. Borisov, R. Bunoiu and G. Cardone, On a waveguide with an infinite number of small windows. C. R. Math. Acad. Sci. Paris, Ser. I 349 (2011) 53-56.

[5] D. Borisov, R. Bunoiu and G. Cardone, Homogenization and asymptotics for a waveguide with an infinite number of closely located small windows. Prob. Math. Anal. 58 (2011) 59-68; J. Math. Sci. 176 (2011) 774-785.

[6] D. Borisov, R. Bunoiu and G. Cardone, Waveguide with non-periodically alternating Dirichlet and Robin conditions: homogenization and asymptotics. Z. Angew. Math. Phys. (ZAMP), DOI 10.1007/s00033-012-0264-2.

[7] D. Borisov and G. Cardone, Homogenization of the planar waveguide with frequently alternating boundary conditions. J. Phys. A, Math. Theor. 42 (2009) 365205.

[8] D. Borisov and G. Cardone, Planar Waveguide with "Twisted" Boundary Conditions: Discrete Spectrum. J. Math. Phys. 52 (2011) 123513.

[9] D. Borisov and G. Cardone, Planar Waveguide with "Twisted" Boundary Conditions: Small Width. J. Math. Phys. 53 (2012) 023503.

[10] D. Borisov, P. Exner, R. Gadyl'shin and D. Krejčiřík, Bound states in weakly deformed strips and layers. Ann. Henri Poincaré 2 (2001) 553-572.

[11] W. Bulla, F. Gesztesy, W. Renger and B. Simon, Weakly coupled bound states in quantum waveguides. Proc. Amer. Math. Soc. 125 (1997) 1487-1495.

[12] G. Cardone, V. Minutolo and S.A. Nazarov, Gaps in the essential spectrum of periodic elastic waveguides. Z. Angew. Math. Mech. 89 (2009) 729-741.

[13] G. Cardone, S.A. Nazarov and C. Perugia, A gap in the continuous spectrum of a cylindrical waveguide with a periodic perturbation of the surface. Math. Nach. 283 (2010) 1222-1244.

[14] G. Cardone, S.A. Nazarov and K. Ruotsalainen, Asymptotics of an eigenvalue in the continuous spectrum of a converging waveguide. Mat. Sb. 203 (2012) 3-32.

[15] G. Cardone, V. Minutolo and S.A. Nazarov, Gaps in the essential spectrum of periodic elastic waveguides. Z. Angew. Math. Mech. 89 (2009) 729-741.

[16] G. Cardone, S.A. Nazarov and C. Perugia, A gap in the continuous spectrum of a cylindrical waveguide with a periodic perturbation of the surface. Math. Nach. 283 (2010) 1222-1244.

[17] P. Duclos and P. Exner, Curvature-induced bound states in quantum waveguides in two and three dimensions. Rev. Math. Phys. 7 (1995) 73-102.

[18] P. Exner and S.A. Vugalter, Bound states in a locally deformed waveguide: the critical case. Lett. Math. Phys. 39 (1997) 59-68.

[19] R.R. Gadyl'shin, On local perturbations of quantum waveguides. (Russian) Teoret. Mat. Fiz. 145 (2005) 358-371; Engl. transl.: Theoret. Math. Phys. 145 (2005) 1678-1690. 
[20] V.V. Grushin, On the eigenvalues of a finitely perturbed Laplace operator in infinite cylindrical domains. Mat. Zametki $\mathbf{7 5}$ (2004) 360-371; Engl. transl.: Math. Notes 75 (2004) 331-340.

[21] D.S. Jones, The eigenvalues of $\nabla^{2} u+\lambda u=0$ when the boundary conditions are given on semi-infinite domains. Proc. Cambridge Philos. Soc. 49 (1953) 668-684.

[22] V.A. Kondratiev, Boundary value problems for elliptic problems in domains with conical or corner points, Trudy Moskov. Matem. Obshch 16 (1967) 209-292. Engl. transl.: Trans. Moscow Math. Soc. 16 (1967) 227-313.

[23] V.G. Maz'ya and B.A. Plamenevskii, On coefficients in asymptotics of solutions of elliptic boundary value problems in a domain with conical points, Math. Nachr. 76 (1977) 29-60; Engl. transl.: Amer. Math. Soc. Transl. 123 (1984) 57-89.

[24] V.G. Maz'ya and B.A. Plamenevskii, Estimates in $L^{p}$ and Hölder classes and the Miranda-Agmon maximum principle for solutions of elliptic boundary value problems in domains with singular points on the boundary. Math. Nachr. 81 (1978) 25-82; Engl. transl.: Amer. Math. Soc. Transl. Ser. 123 (1984) 1-56.

[25] V.G. Maz'ya, S.A. Nazarov and B.A. Plamenevskij, Boris Asymptotic theory of elliptic boundary value problems in singularly perturbed domains II, Translated from the German by Plamenevskij. Operator Theory: Advances and Applications. Birkhäuser Verlag, Basel 112 (2000).

[26] S.A. Nazarov, Two-term asymptotics of solutions of spectral problems with singular perturbations, Mat. sbornik. 178 (1991) 291-320; Engl. transl.: Math. USSR. Sbornik. 69 (1991) 307-340.

[27] S.A. Nazarov, Discrete spectrum of cranked, branchy and periodic waveguides, Algebra i analiz 23 (2011) 206-247; Engl. transl.: St. Petersburg Math. J. 23 (2011).

[28] S.A. Nazarov and B.A. Plamenevsky, Elliptic problems in domains with piecewise smooth boundaries. Nauka, Moscow (1991); Engl. transl.: Elliptic problems in domains with piecewise smooth boundaries. Walter de Gruyter, Berlin, New York (1994). 